\title{
Molecular description of meningeal solitary fibrous tumors/hemangiopericytomas compared to meningiomas: two completely separate entities
}

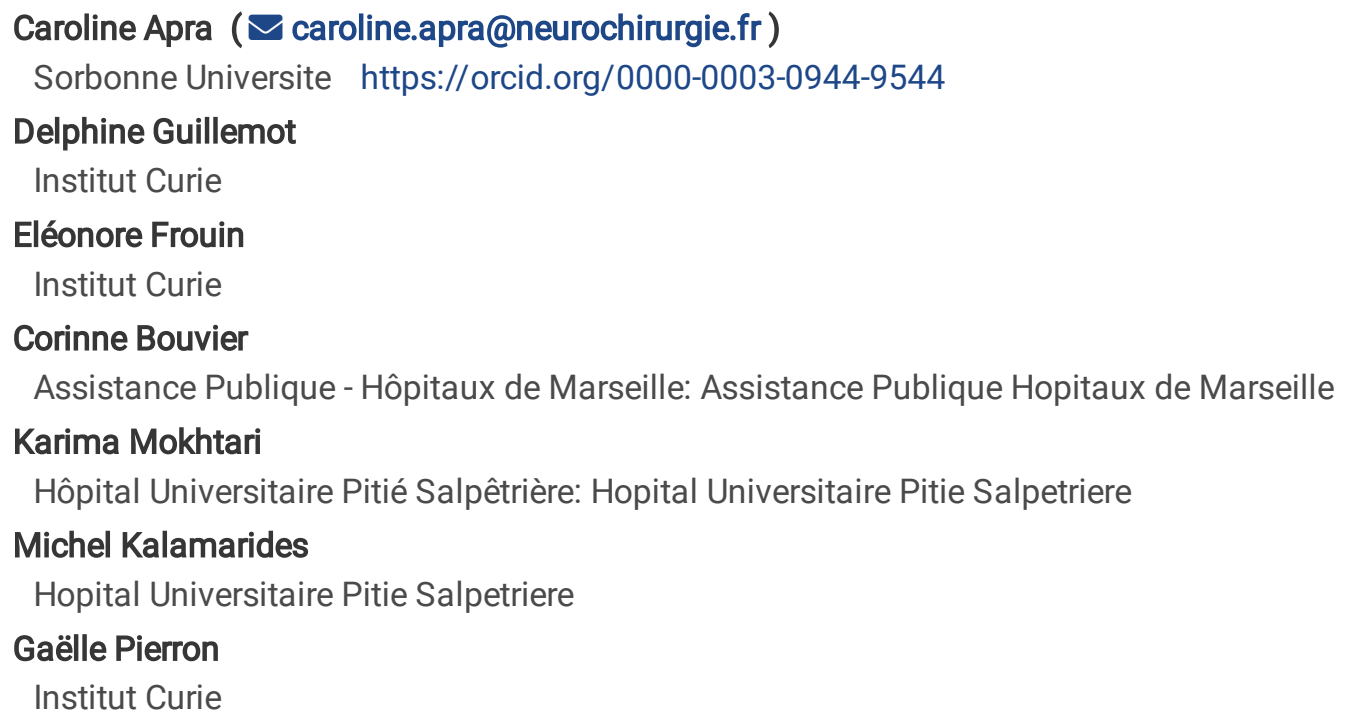

\section{Research Article}

Keywords: meninges, TP53, solitary fibrous tumor, hemangiopericytoma, meningioma, NAB2-STAT6

Posted Date: August 14th, 2021

DOI: https://doi.org/10.21203/rs.3.rs-642486/v1

License: (c) (1) This work is licensed under a Creative Commons Attribution 4.0 International License. Read Full License

Version of Record: A version of this preprint was published at Journal of Neuro-Oncology on August 20th, 2021. See the published version at https://doi.org/10.1007/s11060-021-03830-7. 


\section{Abstract}

Introduction: Meningeal solitary fibrous tumors (SFT), like all SFT, are defined by NAB2-STAT6 fusion and share clinicopathologic similarities with meningiomas, the most frequent meningeal tumors. Our aim is to establish the molecular identity of meningeal SFT and seek molecular prognostic factors.

Methods: RNA sequencing and whole exome sequencing (WES) were performed in STAT6-positive SFT and grade 2-3 meningiomas, and data concerning other soft tissues tumors was obtained from the local database. Uniform manifold approximation and projection (UMAP), individual gene expression and Gene Set Enrichment Analysis (GSEA) were performed.

Results: RNA clustering shows that SFT share a common molecular signature, different from any other type of tumoral tissue. Meningeal SFT aggregate with other SFT, with no clinical or histological subgroup. Comparison of genes expressions suggests significant over-expressions of ZIC2, ZIC3, ZIC5, GABBR2, TP53 in CNS-SFT. The pathogenic TP53 c.743G > T variant, previously undescribed in SFT, was found in one sample of meningeal SFT during malignant progression.

Conclusions: Meningeal SFT are molecular counterparts of extra-meningeal SFT, completely separate from meningiomas. They might develop from the same tissues and benefit from the same treatments as SFT.

\section{Introduction}

Solitary Fibrous Tumors (SFT) are fibroblastic tumors characterized by a prominent branching staghorn vasculature. Most are indolent but recurrences and metastases can occur after several years. Recent multivariate risk models including clinicopathological criteria in addition to the benign/malignant distinction significantly improved prognostication $[1,2]$. Meningeal or central nervous system Solitary Fibrous Tumors (CNS-SFT), formerly also known as hemangiopericytomas [3,4], share the same molecular signature as SFT that develop in other organs, the NAB2-STAT6 fusion [5], and are now classified in grades 1,2 and 3 [3]. However, CNS-SFT are clinically and radiologically very close to meningiomas, which represent the most frequent meningeal tumor type, and resemble in particular grade 2-3 meningiomas [6]. Some authors also suggest that both tumor types share the same cell of origin, prostaglandin-D2-synthase immune-positive cells [7,8]. Like meningiomas, low grade CNS-SFT can transform into malignant CNS-SFT [9]. The entity of CNS-SFT/hemangiopericytomas is a recent group based on histological and molecular patterns [3], and knowledge about the molecular specificities of these tumors is still scarce. Moreover, the clinical course of CNSSFT is varied and unpredictable, which makes it difficult to tailor adjuvant treatments after surgery: up to $10 \%$ of patients develop metastases and $50 \%$ recur within 10 years, even in grade 1 cases $[9,10]$.

It has been shown that all SFT carry the NAB2-STAT6 fusion [5], which is necessary to establish the diagnosis, via the nuclear STAT6 immunostaining [11]. Different types of fusions have been described in SFT in general, and the commonest fusions found in CNS-SFT lead to a protein with a short truncated STAT6, adding only the transcription activating domain to NAB2 [5]. Other CNS-SFT NAB2-STAT6 fusions identified with the progressively available primers include different forms of truncated STAT6. Fusions leading to a short truncated STAT6 are associated with a higher rate of high grade SFT than other fusion types found in extra-meningeal SFT, but the association with clinical prognosis is not clearly established $[12,13]$. In CNS-SFT, those fusions (exon6-exon16/17) are significatively more frequent in grades 2-3 tumors but are not associated with a worse overall or progression-free survival[14]. Thus, until now, the type of fusion is not used as prognostic factor in clinical practice.

Molecular specificities predicting a worse outcome in extra-meningeal SFT include TERT promoter mutations, in hotspots $-124 \mathrm{C}>\mathrm{T}$ and $146 \mathrm{C}>\mathrm{T}$ [14] like in meningiomas, gliomas or medulloblastomas [15,16], but those mutations lack prognostic value in CNSSFT specifically, for both metastatic development and survival[14]. TP53 mutations are associated with a shorter progression-free survival in all SFT [17-22]. In addition, CDKN2AB/p16 locus homozygous deletions are found in $25 \%$ of recurrent CNS-SFT [23]. The molecular comparison between one gluteal grade 2 SFT and its associated malignant cerebral metastasis showed that two pathogenic mutations arised: TP53 exon $4 \mathrm{c.313G}>\mathrm{T}$, a known oncogenic variant inducing TP53 overexpression, and APAF1 exon $12 \mathrm{c} .1669 \mathrm{C}>\mathrm{T}$, causing the loss of APAF1 expression and the decrease of cell apoptosis along the increase of cell migration and proliferation in vitro [17]. These molecular markers are not used in clinical practice yet and need to be confirmed. Moreover, no other molecular study was performed in evolving SFT, such as the primary tumor and metastasis, or the benign tumor and its malignant transformation. 
In order to better describe CNS-SFT molecular signature, we compared them to grade 2-3 meningiomas and to extra-meningeal SFT, based on RNA sequencing profile. In order to identify other prognostic molecular factors, we correlated clinical data to this analysis, and performed whole exome sequencing in samples from patients with recurrent transforming CNS-SFT. Given the rarity of these tumors, the molecular identity of CNS-SFT will have a direct impact on the therapeutics proposed to the patients, orientating either toward a meningioma-like treatment, or toward a SFT treatment.

\section{Materials And Methods}

\subsection{Patients and samples}

Frozen tumors samples were collected from patients operated at the Pitié Salpêtrière hospital (Paris, France) between 2004 and 2018 for grade 1 or 3 CNS-SFT, including patients with a pair of primitive grade 1 and a recurrent grade 3 CNS-SFT, as already described [9] (cf.sup. table1). All histological samples were immunopositive for STAT6 nuclear staining and grading was reassessed according to the 2016 WHO classification $[3,24]$. Clinical data were collected in the medical file.

Meningioma frozen samples were provided by APHM Biobank (CRB-TBM authorization AC2018-31053; CRB BB-0033-00097)[25]. RNA sequencing data concerning other types of tumors was obtained from the local Institut Curie database for SFT and meningiomas samples, among a larger collection of soft tissues tumors (AFNOR NF S 96900 2009/33837B; data available on demand). RNA sequencing allowed identification of NAB2-STAT6 fusion in all SFT samples.

All patients were informed and gave written consent for the anonymized used of samples for molecular analysis, according to the French Ethic Law (AC-2013-1962).

\subsection{Molecular analyses}

Total RNA was extracted from frozen tumor tissues using Trizol-chloroform method (Invitrogen). The RNA integrity was verified using TapeStation 4200 (Agilent Technologies). RNA sequencing was performed with TruSeq Stranded mRNA Prep kit (Illumina) according to the manufacturer's protocol, and libraries sequenced with the illumina NextSeq500 pair-end 150pb.

Gene expression values were estimated from the RNA-seq data using Salmon (v0.13.1) in TPM (Transcript Per Million) The pairedend transcriptome sequencing reads were aligned to the human reference genome (GRCh37/hg19) with StarAligner. We analyzed expressed mutation with HaplotypeCaller and Mutect2 from GATK4). Gene fusion were analysed from FASTQ data fusion finder tools (FusionMap from Oshell (v10.0.1.50), Starfusion (v2.5), FusionCatcher (v1.0), Defuse (v0.6.2)).

Gene expression datas allow a comparison between samples by supervised clustering using distance calculation and Pearson correlation. With the list of genes of $5 \%$ internal quantile range (IQR) variation, we looked for enrichment of pathways (GSEA, Gene Set Enrichment Analysis open access software GSEA, Broad Institute, USA) with gene sets v.7.1 and genes annotations Human_Illumina_HumanHT_12_v3_MSigDB.v7.1.chip. Using 100 permutations, we analyzed gene sets with a False Discovery Rate $<25 \%$. Literature analysis was performed on several gene databases $[26,27]$. GSEA was performed for SFT versus meningiomas and for SFT subgroups identified in the unsupervised RNA clustering.

We compared gene expression of SFT and meningiomas within a panel of well-characterized samples: small round cells tumors, soft tissues sarcoma (with or without fusion transcript), and, brain tumors corresponding to 258 cases in total. A set of 5,000 most variable transcripts was used to perform a principal component analysis (PCA). The first 50 components of this PCA were then selected to implement 2D UMAP representation. Aggregation of samples allows identification of a characteristic expression profile between different groups proposed (SFT vs meningiomas). VarSome prediction was used to identify pathogenic or likely pathogenic mutations [28].

\section{Results}

3.1 Patients and samples 
The whole exome sequencing (WES) analysis included 5 pairs of recurrent grade 1-3 CNS-SFT, among which 3 pairs could be interpreted (cf.sup.table1). The two remaining pairs were paraffin-embedded samples and quality control of WES was not satisfying, hindering further interpretation.

The RNA sequencing analysis included 7 grade 1 CNS-SFT, 6 grade 3 CNS-SFT (including 2 grade 1-3 pairs), 3 extra-meningeal grade 1 SFT, 9 extra-meningeal grade 3 SFT, 14 grade 2 meningiomas and 11 grade 3 meningiomas (cf.sup.table2).

\subsection{Molecular analyses}

The results of WES comparison between the primitive and the malignant CNS-SFT recurrence are shown in supplementary table 3. According to VarSome predictors, only one pathogenic variant was identified during histological malignant progression, in one case, the pathogenic TP53 c.743G >T, and RNA sequencing showed that TP53 was indeed expressed in the sample.

Analysis of RNA expression confirms that all SFT express NAB2-STAT6 fusions. To evaluate global expression profiles of our tumoral entities, a first step of UMAP analysis was performed. Uniform manifold approximation and projection (UMAP) is a scalable and efficient dimension reduction algorithm that performs competitively among state-of-the-art methods such as t-SNE [29], and widely applied for unsupervised clustering. So it can be used as an effective preprocessing step to boost the performance of density based clustering [30] (cf.fig1.a). Here our RNAseq of CNS-SFT, SFT and meningiomas were compared to a larger group of varied tumoral samples (258 in total), which shows that all SFT, independently of their localization, aggregate and that CNSSFT are closer from any SFT than from any other type of tumors (cf.fig1.a highlighted by colored circles). This pattern suggests that all SFT share a common molecular signature, different from any other type of tumoral tissue.

When focusing on the subgroup of soft tissue tumors, unsupervised RNA sequencing clustering shows that SFT from all localizations aggregate together in a cluster separate from meningiomas and from other types of soft tissue tumors (cf.sup.fig1). Zooming only on SFT and meningiomas, we show that CNS-SFT aggregate with extra-meningeal SFT and completely separately from meningiomas, without a single exception. A supervised clustering based on the 50 most differentially expressed genes show clearly specific and distinct expression pattern on the heatmap (cf.fig1.b). Among SFT, CNS-SFT do not constitute a separate cluster and no subgroup can be defined based on clinical criteria or grading, except a tendency for older patients to gather on the left part of the SFT cluster. The two pairs of recurrent CNS-SFT show that the primitive CNS-SFT and the malignant recurrence share a similar transcriptome, despite the histological transformation. However, both pairs do not share close transcriptomes (cf.fig1.b). Therefore, in our collection, there is no argument for a molecular difference between CNS-SFT and SFT.

The analysis of molecular pathways affected by the most weighted genes underlying the common SFT identity revealed no relevant oncological pathway. We also used GSEA analysis for older patients ( $>65$ year-old) whose samples RNA clustered separately from younger patients and found that 5 gene sets were gene sets are potential candidates:

GO_NEGATIVE_REGULATION_OF_PEPTIDYL_THREONINE_PHOSPHORYLATION, GO_INTRACELLULAR_STEROL_TRANSPORT, GO_SERINE_FAMILY_AMINO_ACID_BIOSYNTHETIC_PROCESS,

GO_TRANSFERASE_ACTIVITY_TRANSFERRING_NITROGENOUS_GROUPS and CHR1P33 (Ensembl 99 Genes in Cytogenetic Band chr1p33). None of them is implicated in potentially oncogenic processes, and the chr1p33 gain has not been reported in any type of cancer.

We also used GSEA to explore pathways that could be affected by SFT development, based on list of 209 genes relevant to meninges physiopathology, for SFT versus meningiomas. We found three potentially oncogenic gene sets, YAP1_DN, usually under-expressed in breast cancer, ESC_V6.5_UP_EARLY.V1_UP, which includes gene implicated in stem cells differentiation in mice, and NAGY_TFTC_COMPONENTS_HUMAN, genes implicated in RNA transcription through histones and chromatin remodeling. None of these pathways is known to be involved in SFT development.

Individual expression of the list of genes relevant in the literature and in the RNA sequencing clustering analysis was compared in SFT versus meningiomas, and in CNS-SFT versus extra-meningeal SFT. We selected 67 genes significantly differentially expressed in SFT versus meningiomas, and 10 genes in CNS-SFT versus extra-meningeal SFT. Based on the literature, the over-expressions of ZIC1, ZIC2, ZIC3, ZIC5, GABBR2, TP53 might potentially be relevant in CNS-SFT (cf.fig1.c). 


\section{Conclusions}

Transcriptome analyses confirm that CNS-SFT are fully part of the SFT group, completely separate from meningiomas. Moreover, they do not seem to present any molecular characteristic that makes them different from extra-meningeal SFT. We know they share common molecular characteristics, with the driving NAB2-STAT6 fusion [5], and we showed that their transcriptomes are also similar. This common molecular identity suggests that a common therapeutic management might be of some relevance. In addition, it may help understand the physiopathology of SFT development, in particular in the CNS. The complete molecular separation between meningiomas and CNS-SFT is not in favor of a common tissue of origin, which needs to be elucidated. It could be a clue to understand why CNS-SFT cause extra-central nervous system metastases more often than other meningeal tumors $[9,31]$.

When questioning what drives the molecular identity of SFT, no relevant gene sets involvement could be identified. There is also no clinically or histologically defined subgroup that could be associated with molecular characteristics, especially the grade of the SFT or its localization. Clustering of older patients with SFT may be an artefact due to aging, associated with a louder background noise. No specific gene set enrichment could illustrate the specificity of this subgroup, which tends to confirm this hypothesis.

In both meningiomas and CNS-SFT, the ZIC family (significantly ZIC2, ZIC3, ZIC5, and not ZIC1 and ZIC4) is over-expressed. They participate in the development of skull and meninges, which is coherent with their expression in meningeal tumors compared to extra-meningeal [32](ref). They are paralogs and the reason why only some of them are significantly over-expressed is not clear. ZIC5 is overexpressed in different types of cancers [33,34] and may contribute to cell proliferation and development of drug resistance. For instance, it is a potential therapeutic target in melanoma, colorectal or non-small cell lung cancer [35].

GABBR2, is over-expressed in some CNS-SFT specifically, and analysis of those patients showed they share no common clinical or histological characteristics. GABBR1, coding for the compulsory heterodimer of GABBR2 [36] is also expressed in this subgroup, making it relevant to consider an increased functional protein (cf.fig.1.c). GABBR1-GABBR2 is a membrane GABA receptor, active in potassium-dependent neuronal signal. Whether targeting GABBR2 as a therapeutic option in CNS-SFT would be relevant is still highly questionable at this point, although it might be possible with VAR2CSA immunotherapy, and it is a target for the treatment of resistant lung adenocarcinomas [37,38].

The only molecular event certainly associated with one case of malignant transformation of CNS-SFT in our series is TP53 variant, as already described in other SFT cases detailed in table 1 [17-22]. The variant we found, heterozygous missense exon 7 c.743G $>\mathrm{T}$, had not been reported yet in SFT, and is pathogenic in several malignant tumors, such as sarcomas, glioblastomas and familial forms of cancers [39]. Although some authors proposed that TP53 mutations are already present in low grades SFT, we know this was not the case in our patients [40], and is therefore not a prognostic factor at the time of the first CNS-SFT occurrence. Taking into account that SFT are macroscopically heterogeneous tumors and grade 1 tumors may well have contained a malignant part not represented in the sample, and that an emerging clone of mutated TP53 may not have been detected for technical reasons, if it was present in small amounts.

In conclusion, CNS-SFT are molecular counterparts of extra-meningeal SFT, completely separate from meningiomas. We identified a new TP53 mutation, c.743G $>$ T, which is associated with malignant CNS-SFT transformation in some cases. Until now, most clinical trials focus on extra-meningeal SFT, excluding intracranial localizations or metastases [41]. Our findings, associated with the histological identification of hemangiopericytomas with CNS-SFT and with previously published molecular data [5], suggests a common therapeutic approach for all types of SFT, regardless of their localization, might be relevant. As already reported in some cases [42], treatments usually used in soft tissues SFT have proved useful in meningeal SFT and could be discussed with general oncologists, rather than using meningiomas treatments in CNS-SFT, with this in mind that the treatment of meningeal localizations may have to be adapted for adequate delivery.

\section{Declarations}

Funding: This work was supported by a grant from the Fondation ARC (PJA 20151203101).

Conflicts of interest: None

Page 5/12 
Availability of data and material: available for academic use on demand

Code availability : on demand

Authors' contributions:

CA: Conceptualization; Formal analysis; Investigation; Writing - original draft

DG: Formal analysis; Investigation; Writing - original draft

EF: Formal analysis; Investigation

CB: Data curation; Writing - review \& editing

KM: Data curation; Writing - review \& editing

MK: Conceptualization; Funding acquisition; Supervision; Writing - review \& editing

GP: Conceptualization; Methodology; Supervision; Validation; Writing - review \& editing

Ethics approval: APHM Biobank (CRB-TBM authorization AC2018-31053; CRB BB-0033-00097), Institut Curie (AFNOR NF S 96900 2009/33837B)

Consent to participate: All patients were informed and gave written consent for the anonymized used of samples for molecular analysis, according to the French Ethic Law (AC-2013-1962).

Consent for publication: All patients were informed and gave written consent for the anonymized used of samples for molecular analysis, according to the French Ethic Law (AC-2013-1962).

Acknowledgements: The authors thank Genosplice for exome sequencing and Exorare for access to molecular data.

\section{References}

[1] W.C. of T.E. Board, Soft Tissue and Bone Tumours, n.d. https://publications.iarc.fr/Book-And-Report-Series/WhoClassification-Of-Tumours/Soft-Tissue-And-Bone-Tumours-2020 (accessed April 12, 2021).

[2] E.G. Demicco, A.M. Griffin, R.A. Gladdy, B.C. Dickson, P.C. Ferguson, C.J. Swallow, J.S. Wunder, W.L. Wang, Comparison of published risk models for prediction of outcome in patients with extrameningeal solitary fibrous tumour, HISTOPATHOLOGY. 75 (2019) 723-737. https://doi.org/10.1111/his.13940.

[3] D.N. Louis, A. Perry, G. Reifenberger, A. von Deimling, D. Figarella-Branger, W.K. Cavenee, H. Ohgaki, O.D. Wiestler, P. Kleihues, D.W. Ellison, The 2016 World Health Organization Classification of Tumors of the Central Nervous System: a summary, Acta Neuropathol. 131 (2016) 803-820. https://doi.org/10.1007/s00401-016-1545-1.

[4] C. Bouvier, P. Métellus, A.M. de Paula, A. Vasiljevic, A. Jouvet, J. Guyotat, K. Mokhtari, P. Varlet, H. Dufour, D. FigarellaBranger, Solitary fibrous tumors and hemangiopericytomas of the meninges: overlapping pathological features and common prognostic factors suggest the same spectrum of tumors, Brain Pathol. 22 (2012) 511-521. https://doi.org/10.1111/j.17503639.2011.00552.x.

[5] D.R. Robinson, Y.-M. Wu, S. Kalyana-Sundaram, X. Cao, R.J. Lonigro, Y.-S. Sung, C.-L. Chen, L. Zhang, R. Wang, F. Su, M.K. Iyer, S. Roychowdhury, J. Siddiqui, K.J. Pienta, L.P. Kunju, M. Talpaz, J.M. Mosquera, S. Singer, S.M. Schuetze, C.R. Antonescu, A.M. Chinnaiyan, Identification of recurrent NAB2-STAT6 gene fusions in solitary fibrous tumor by integrative sequencing, Nat Genet. 45 (2013) 180-185. https://doi.org/10.1038/ng.2509.

[6] S. Trabelsi, N. Mama, M. Chourabi, M.H. Mastouri, M. Ladib, S. Popov, A. Burford, M. Mokni, K. Tlili, H. Krifa, C. Jones, M.T. Yacoubi, A. Saad, D.H.-B. Brahim, Meningeal Hemangiopericytomas and Meningomas: a Comparative Immunohistochemical and 
Genetic Study, Asian Pac. J. Cancer Prev. 16 (2015) 6871-6876.

[7] M. Peyre, C. Salaud, E. Clermont-Taranchon, M. Niwa-Kawakita, S. Goutagny, C. Mawrin, M. Giovannini, M. Kalamarides, PDGF activation in PGDS-positive arachnoid cells induces meningioma formation in mice promoting tumor progression in combination with Nf2 and Cdkn2ab loss, Oncotarget. 6 (2015) 32713-32722. https://doi.org/10.18632/oncotarget.5296.

[8] M. Kawashima, S.O. Suzuki, T. Yamashima, M. Fukui, T. Iwaki, Prostaglandin D synthase (beta-trace) in meningeal hemangiopericytoma, Mod. Pathol. 14 (2001) 197-201. https://doi.org/10.1038/modpathol.3880285.

[9] C. Apra, K. Mokhtari, P. Cornu, M. Peyre, M. Kalamarides, Intracranial solitary fibrous tumors/hemangiopericytomas: first report of malignant progression, J. Neurosurg. (2017) 1-6. https://doi.org/10.3171/2017.1.JNS162593.

[10] O. Damodaran, P. Robbins, N. Knuckey, M. Bynevelt, G. Wong, G. Lee, Primary intracranial haemangiopericytoma: Comparison of survival outcomes and metastatic potential in WHO grade II and III variants, Journal of Clinical Neuroscience. 21 (2014) 1310-1314. https://doi.org/10.1016/j.jocn.2013.11.026.

[11] L. Schweizer, C. Koelsche, F. Sahm, R.M. Piro, D. Capper, D.E. Reuss, S. Pusch, A. Habel, J. Meyer, T. Göck, D.T.W. Jones, C. Mawrin, J. Schittenhelm, A. Becker, S. Heim, M. Simon, C. Herold-Mende, G. Mechtersheimer, W. Paulus, R. König, O.D. Wiestler, S.M. Pfister, A. von Deimling, Meningeal hemangiopericytoma and solitary fibrous tumors carry the NAB2-STAT6 fusion and can be diagnosed by nuclear expression of STAT6 protein, Acta Neuropathol. 125 (2013) 651-658. https://doi.org/10.1007/s00401-0131117-6.

[12] S. Yuzawa, H. Nishihara, L. Wang, M. Tsuda, T. Kimura, M. Tanino, S. Tanaka, Analysis of NAB2-STAT6 Gene Fusion in 17 Cases of Meningeal Solitary Fibrous Tumor/Hemangiopericytoma: Review of the Literature, Am. J. Surg. Pathol. (2016). https://doi.org/10.1097/PAS.0000000000000625.

[13] S. Barthelmeß, H. Geddert, C. Boltze, E.A. Moskalev, M. Bieg, H. Sirbu, B. Brors, S. Wiemann, A. Hartmann, A. Agaimy, F. Haller, Solitary fibrous tumors/hemangiopericytomas with different variants of the NAB2-STAT6 gene fusion are characterized by specific histomorphology and distinct clinicopathological features, Am. J. Pathol. 184 (2014) 1209-1218.

https://doi.org/10.1016/j.ajpath.2013.12.016.

[14] R. Vogels, N. Macagno, K. Griewank, P. Groenen, M. Verdijk, J. Fonville, B. Kusters, French CNS SFT/HPC Consortium, Dutch CNS SFT/HPC Consortium, D. Figarella-Branger, P. Wesseling, C. Bouvier, U. Flucke, Prognostic significance of NAB2-STAT6 fusion variants and TERT promotor mutations in solitary fibrous tumors/hemangiopericytomas of the CNS: not (yet) clear, Acta Neuropathol. 137 (2019) 679-682. https://doi.org/10.1007/s00401-019-01968-3.

[15] C. Koelsche, F. Sahm, D. Capper, D. Reuss, D. Sturm, D.T.W. Jones, M. Kool, P.A. Northcott, B. Wiestler, K. Böhmer, J. Meyer, C. Mawrin, C. Hartmann, M. Mittelbronn, M. Platten, B. Brokinkel, M. Seiz, C. Herold-Mende, A. Unterberg, J. Schittenhelm, M. Weller, S. Pfister, W. Wick, A. Korshunov, A. von Deimling, Distribution of TERT promoter mutations in pediatric and adult tumors of the nervous system, Acta Neuropathol. 126 (2013) 907-915. https://doi.org/10.1007/s00401-013-1195-5.

[16] C. Mirian, A.K. Duun-Henriksen, T. Juratli, F. Sahm, S. Spiegl-Kreinecker, M. Peyre, A. Biczok, J.-C. Tonn, S. Goutagny, L. Bertero, A.D. Maier, M. Møller Pedersen, I. Law, H. Broholm, D.P. Cahill, P. Brastianos, L. Poulsgaard, K. Fugleholm, M. Ziebell, T. Munch, T. Mathiesen, Poor prognosis associated with TERT gene alterations in meningioma is independent of the WHO classification: an individual patient data meta-analysis, J. Neurol. Neurosurg. Psychiatry. 91 (2020) 378-387. https://doi.org/10.1136/jnnp-2019-322257.

[17] H.K. Park, D.B. Yu, M. Sung, E. Oh, M. Kim, J.-Y. Song, M.-S. Lee, K. Jung, K.-W. Noh, S. An, K. Song, D.-H. Nam, Y.J. Kim, Y.-L. Choi, Molecular changes in solitary fibrous tumor progression, J. Mol. Med. 97 (2019) 1413-1425. https://doi.org/10.1007/s00109-019-01815-8.

[18] Y. Morimitsu, M. Nakajima, M. Hisaoka, H. Hashimoto, Extrapleural solitary fibrous tumor: clinicopathologic study of 17 cases and molecular analysis of the p53 pathway, APMIS. 108 (2000) 617-625. https://doi.org/10.1034/j.1600-0463.2000.d01- 
105.x.

[19] M.M. Subramaniam, X.Y. Lim, K. Venkateswaran, C.S. Shuen, R. Soong, F. Petersson, Dedifferentiated solitary fibrous tumour of the nasal cavity: the first case reported with molecular characterization of a TP53 mutation, Histopathology. 59 (2011) 1269-1274. https://doi.org/10.1111/j.1365-2559.2011.03997.x.

[20] K. Akaike, A. Kurisaki-Arakawa, K. Hara, Y. Suehara, T. Takagi, K. Mitani, K. Kaneko, T. Yao, T. Saito, Distinct clinicopathological features of NAB2-STAT6 fusion gene variants in solitary fibrous tumor with emphasis on the acquisition of highly malignant potential, Hum. Pathol. 46 (2015) 347-356. https://doi.org/10.1016/j.humpath.2014.11.018.

[21] I. Machado, G.N. Morales, J. Cruz, J. Lavernia, F. Giner, S. Navarro, A. Ferrandez, A. Llombart-Bosch, Solitary fibrous tumor: a case series identifying pathological adverse factors-implications for risk stratification and classification, Virchows Arch. 476 (2020) 597-607. https://doi.org/10.1007/s00428-019-02660-3.

[22] A. Kurisaki-Arakawa, K. Akaike, K. Hara, A. Arakawa, M. Takahashi, K. Mitani, T. Yao, T. Saito, A case of dedifferentiated solitary fibrous tumor in the pelvis with TP53 mutation, Virchows Arch. 465 (2014) 615-621. https://doi.org/10.1007/s00428-0141625-3.

[23] Y. Ono, K. Ueki, J.T. Joseph, D.N. Louis, Homozygous deletions of the CDKN2/p16 gene in dural hemangiopericytomas, Acta Neuropathol. 91 (1996) 221-225.

[24] L.A. Doyle, M. Vivero, C.D. Fletcher, F. Mertens, J.L. Hornick, Nuclear expression of STAT6 distinguishes solitary fibrous tumor from histologic mimics, Mod. Pathol. 27 (2014) 390-395. https://doi.org/10.1038/modpathol.2013.164.

[25] E. Dougy, D. Figarella-Branger, P.-E. Morange, A.D. Sandre-Giovannoli, B. Lacarelle, K. Bertaux, K. Pedeillier, N. Saut, C. JiguetJiglaire, S. Tong, K. Achache, The Assistance Publique Hôpitaux de Marseille's Biobank, Open Journal of Bioresources. 7 (2020) 8. https://doi.org/10.5334/ojb.63.

[26] GeneCards - Human Genes | Gene Database | Gene Search, (n.d.). https://www.genecards.org/ (accessed March 31, 2021).

[27] Home - Gene - NCBI, (n.d.). https://www.ncbi.nlm.nih.gov/gene (accessed March 29, 2020).

[28] C. Kopanos, V. Tsiolkas, A. Kouris, C.E. Chapple, M. Albarca Aguilera, R. Meyer, A. Massouras, VarSome: the human genomic variant search engine, Bioinformatics. 35 (2019) 1978-1980. https://doi.org/10.1093/bioinformatics/bty897.

[29] F. de A. e Lima, UMAP clustering in Python, Poissonisfish. (2020). https://poissonisfish.com/2020/11/14/umap-clusteringin-python/ (accessed March 31, 2021).

[30] L. McInnes, J. Healy, J. Melville, UMAP: Uniform Manifold Approximation and Projection for Dimension Reduction, ArXiv:1802.03426 [Cs, Stat]. (2020). http://arxiv.org/abs/1802.03426 (accessed March 31, 2021).

[31] H. Dufour, P. Métellus, S. Fuentes, X. Murracciole, J. Régis, D. Figarella-Branger, F. Grisoli, Meningeal hemangiopericytoma: a retrospective study of 21 patients with special review of postoperative external radiotherapy, Neurosurgery. 48 (2001) 756-762; discussion 762-763.

[32] J. Aruga, K.J. Millen, ZIC1 Function in Normal Cerebellar Development and Human Developmental Pathology, Adv. Exp. Med. Biol. 1046 (2018) 249-268. https://doi.org/10.1007/978-981-10-7311-3_13.

[33] R. Satow, S. Inagaki, C. Kato, M. Shimozawa, K. Fukami, Identification of zinc finger protein of the cerebellum 5 as a survival factor of prostate and colorectal cancer cells, Cancer Sci. 108 (2017) 2405-2412. https://doi.org/10.1111/cas.13419.

[34] R. Houtmeyers, J. Souopgui, S. Tejpar, Deregulation of ZIC Family Members in Oncogenesis, in: J. Aruga (Ed.), Zic Family: Evolution, Development and Disease, Springer, Singapore, 2018: pp. 329-338. https://doi.org/10.1007/978-981-10-7311-3_16. 
[35] R. Satow, T. Nakamura, C. Kato, M. Endo, M. Tamura, R. Batori, S. Tomura, Y. Murayama, K. Fukami, ZIC5 drives melanoma aggressiveness by PDGFD-mediated activation of FAK and STAT3, Cancer Res. (2016). https://doi.org/10.1158/0008-5472.CAN16-0991.

[36] GABBR2 Gene - GeneCards | GABR2 Protein | GABR2 Antibody, (n.d.). https://www.genecards.org/cgi-bin/carddisp.pl? gene=GABBR2 (accessed August 30, 2020).

[37] M.Ø. Agerbæk, S.R. Bang-Christensen, M.-H. Yang, T.M. Clausen, M.A. Pereira, S. Sharma, S.B. Ditlev, M.A. Nielsen, S. Choudhary, T. Gustavsson, P.H. Sorensen, T. Meyer, D. Propper, J. Shamash, T.G. Theander, A. Aicher, M. Daugaard, C. Heeschen, A. Salanti, The VAR2CSA malaria protein efficiently retrieves circulating tumor cells in an EpCAM-independent manner, Nat Commun. 9 (2018) 3279. https://doi.org/10.1038/s41467-018-05793-2.

[38] S.R. Bang-Christensen, R.S. Pedersen, M.A. Pereira, T.M. Clausen, C. Løppke, N.T. Sand, T.D. Ahrens, A.M. Jørgensen, Y.C. Lim, L. Goksøyr, S. Choudhary, T. Gustavsson, R. Dagil, M. Daugaard, A.F. Sander, M.H. Torp, M. Søgaard, T.G. Theander, O. Østrup, U. Lassen, P. Hamerlik, A. Salanti, M.Ø. Agerbæk, Capture and Detection of Circulating Glioma Cells Using the Recombinant VAR2CSA Malaria Protein, Cells. 8 (2019). https://doi.org/10.3390/cells8090998.

[39] NM_000546 c.743G>T SNV | hg19, (n.d.). https://varsome.com/variant/hg19/NM_000546\%20c.743G\%3ET (accessed September 1, 2020).

[40] G.P. Dagrada, R.D. Spagnuolo, V. Mauro, E. Tamborini, L. Cesana, A. Gronchi, S. Stacchiotti, M.A. Pierotti, T. Negri, S. Pilotti, Solitary fibrous tumors: loss of chimeric protein expression and genomic instability mark dedifferentiation, Mod. Pathol. 28 (2015) 1074-1083. https://doi.org/10.1038/modpathol.2015.70.

[41] Search of: solitary fibrous tumor - List Results - ClinicalTrials.gov, (n.d.). https://clinicaltrials.gov/ct2/results? recrs=\&cond=solitary+fibrous+tumor\&term $=\&$ cntry $=\&$ state=\&city=\&dist= (accessed March 31, 2021).

[42] C. Apra, A. Alentorn, K. Mokhtari, M. Kalamarides, M. Sanson, Pazopanib efficacy in recurrent central nervous system hemangiopericytomas, J. Neurooncol. 139 (2018) 369-372. https://doi.org/10.1007/s11060-018-2870-0.

\section{Table}




\begin{tabular}{|c|c|c|c|c|c|c|c|c|}
\hline Reference & $\begin{array}{l}\text { Reported } \\
\text { TP53 } \\
\text { variant }\end{array}$ & Position & Exon & $\begin{array}{l}\text { Missense } \\
\text { protein }\end{array}$ & Prediction & $\begin{array}{l}\text { Number of } \\
\text { publications } \\
\text { (VarSome) }\end{array}$ & SFT grade & $\begin{array}{l}\text { SFT } \\
\text { localization }\end{array}$ \\
\hline Park, 2019 & c. $313 G>T$ & 7579374 & 4 & p.Gly105Cys & Pathogenic & 13 & Malignant & $\begin{array}{l}\text { Cerebral } \\
\text { metastasis }\end{array}$ \\
\hline Kurisaki, 2014 & c. $473 G>A$ & 7577069 & 4 & p.Arg158His & $\begin{array}{l}\text { Uncertain } \\
\text { significance }\end{array}$ & 123 & Malignant & Pelvis \\
\hline $\begin{array}{l}\text { Morimitsu, } \\
2000\end{array}$ & c. $481 \mathrm{G}>\mathrm{A}$ & 7578449 & 5 & p.Ala161Thr & $\begin{array}{l}\text { Likely } \\
\text { Pathogenic }\end{array}$ & 80 & Benign & Peritoneum \\
\hline $\begin{array}{l}\text { Subramaniam, } \\
2011\end{array}$ & c. $711 \mathrm{G}>\mathrm{A}$ & 7577570 & 7 & p.Met237lle & Pathogenic & 163 & Malignant & $\begin{array}{l}\text { Nasal } \\
\text { cavity }\end{array}$ \\
\hline Park, 2019 & c. $742 C>T$ & 7577539 & 7 & p.Arg248Trp & Pathogenic & & Malignant & $\mathrm{N} / \mathrm{A}$ \\
\hline This report & c. $743 \mathrm{G}>\mathrm{T}$ & 7577538 & 7 & p.Arg248Leu & Pathogenic & 147 & Malignant & Meninges \\
\hline Park, 2019 & c. $818 \mathrm{G}>\mathrm{A}$ & 7577120 & 8 & p.Arg273His & Pathogenic & 616 & Malignant & $\mathrm{N} / \mathrm{A}$ \\
\hline Park, 2019 & c. $832 C>T$ & 7576897 & 9 & p.GIn278Ter & Pathogenic & 242 & Malignant & $\mathrm{N} / \mathrm{A}$ \\
\hline $\begin{array}{l}\text { Machado, } \\
2019\end{array}$ & $\begin{array}{l}5 \\
\text { variants }\end{array}$ & - & $5,6,7,8$ & - & - & - & $\begin{array}{l}\text { Mostly } \\
\text { malignant }\end{array}$ & $\begin{array}{l}\text { Soft } \\
\text { tissues }\end{array}$ \\
\hline Akaike, 2015 & $\begin{array}{l}2 \\
\text { variants }\end{array}$ & - & - & - & - & - & $\begin{array}{l}\text { Mostly } \\
\text { malignant }\end{array}$ & $\mathrm{N} / \mathrm{A}$ \\
\hline
\end{tabular}

\section{Figures}




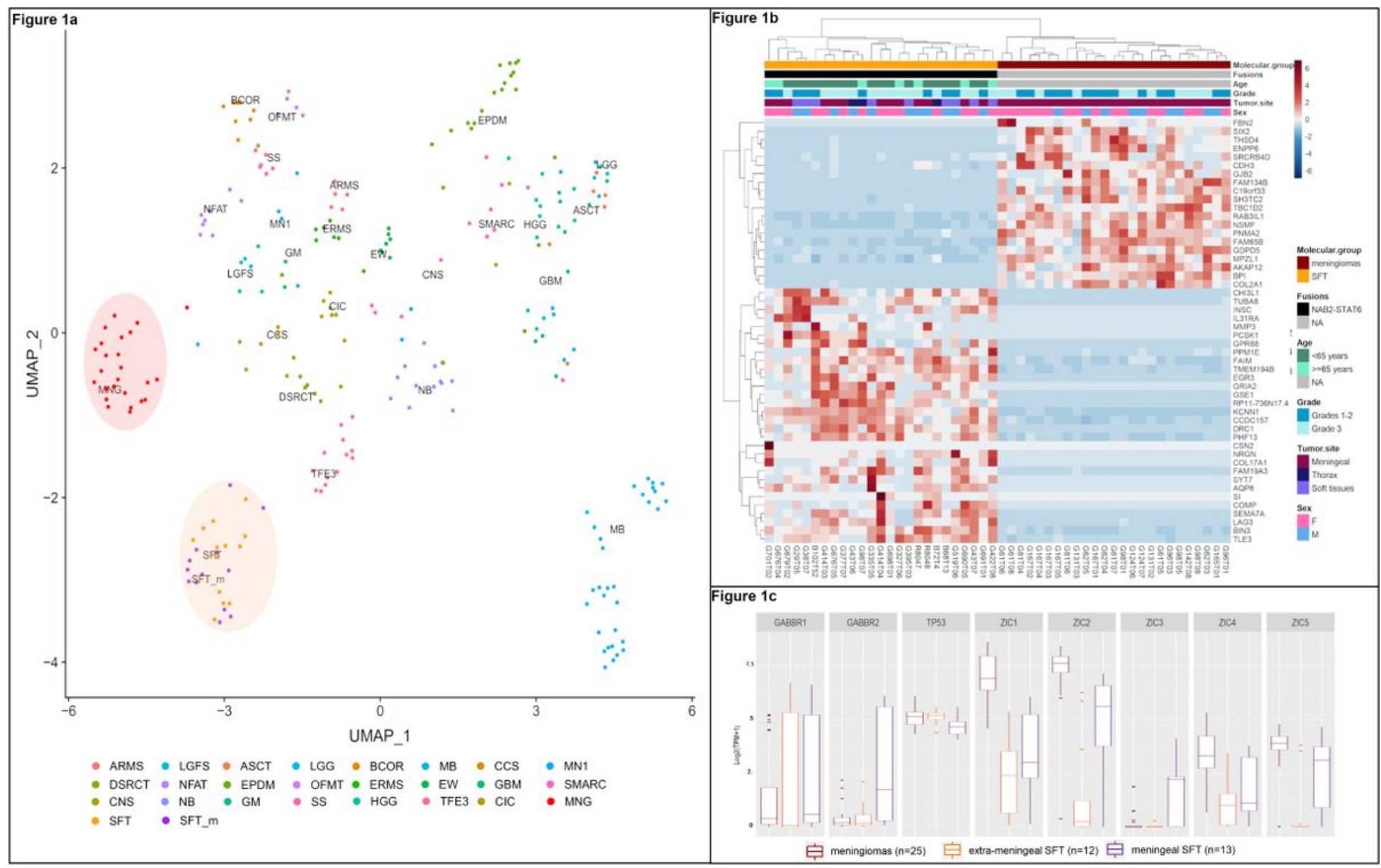

\section{Figure 1}

RNA sequencing analyses for solitary fibrous tumors (SFT), meningiomas, and different types of tumors. 1a. UMAP shows SFT (highlighted within a purple circle) cluster independently from all other tumor types such as meningiomas (red circle), and other tumoral entities of the panel of RNAseq used. Two 1st dimensions are used to performed the representation (UMAP_1 vs UMAP_2) (Panel of used samples is composed of ARMS : Alveolar Rhabdomyosarcoma ( $n=5)$, ERMS : Embryonal Rhabdomyosarcoma $(n=7)$, ASCT : Astrocytome $(n=6)$, BCOR : BCOR-CCNB3 sarcoma $(n=8)$, CCS : Clear cell sarcoma $(n=6)$, CIC : CIC-fused sarcoma $(n=11)$, DSRCT : Desmoplastic Small Round Cell Tumor $(n=6)$, EPDM : Ependymoma $(n=21)$, EW : Ewing Sarcoma $(n=11), G B M$ : Glioblastoma ( $n=7)$, GM : Glioma $(n=3)$, HGG : High Grade Glioma $(n=13)$, LGFS : Low-Grade Fibromyxoid Sarcoma $(n=6)$, LGG : Low Grade Glioma ( $n=5)$, MB : Medulloblastoma ( $n=37)$, MN1 : CNS HGNET MN1-fused $(n=6)$, MNG : Meningiomas $(n=25)$, NB : Neuroblastoma $(n=13)$, NFAT : FET NFATc2 fused $(n=8)$, OFMT : Ossifying Fibromyxoid Tumors $(n=5)$, SMARC : Baf-deficient sarcoma $(n=12)$, SS : Synovialsarcoma $(n=7)$, TFE3 : Alveolar soft part sarcoma $(n=12)$, CNS : Other CNS tumor $(n=5), S F T$ : Solitary Fibrous Tumors $(n=12)$, SFT_m : Solitary Fibrous Tumors $(n=13)$. 1b. Supervised Pearson RNA sequencing for SFT and meningiomas samples with clinical data (histological grade, localization of the tumor, sex, age). SFT from all localizations aggregate completely separately from meningiomas. Among SFT, there is no subgroup clustering, in particular no clustering based on the histological grade or localization. There is a tendency for older patients to aggregate, however, no common molecular pathway could be identified and this might be due to molecular aging artefacts. Meningiomas are figured in red and SFT in orange. 1c. Expression of eight relevant genes in subgroups of tumors. Gene expression was analyzed for the most weighted genes involved in SFT clustering based on RNA sequencing analysis, and for 209 genes implicated in meninges physiopathology. Significantly differentially expressed genes include the ZIC family genes, TP53 and GABBR 1 and 2. Gene expression represented in boxplots (median, interquartile range) for each gene, with each tumor represented by one point on the vertical axis, in log2 scale. Red: meningiomas; orange: extra-meningeal solitary fibrous tumors; purple: meningeal solitary fibrous tumors. From left to right: GABBR2 is over-expressed in CNS-SFT with a subgroup of six patients clearly over-expressing the gene. They share no common clinical or histological trait. GABBR2 compulsory heterodimer GABBR1 is also expressed in the samples, making it possible to consider functional over-expression of the gene. (TPM median is 0.5 for CNS-SFT and 0.1 for SFT and 2.4 for CNS-SFT and 0.1 for 
SFT for GABBR1 and GABBR2). -TP53 is under-expressed in CNS-SFT, significantly compared to extra-meningeal SFT. (TPM median is 24.3 for CNS-SFT and 35.3 for SFT). Mutation of TP53 may lead in over- or under-expression. In our mutated c.743G>T sample, TP53 was expressed at a median level (TPM value 25.7). -Paralogs ZIC2,3,5 are significantly over-expressed in CNS-SFT compared to SFT, and ZIC1,4 are non-significantly over-expressed. The ZIC genes are involved in cranio-facial development and ZIC5 specifically is implicated in many tumor types growth. (TPM median is 47, 3.6 and 7.6 for CNS-SFT and $0.2,0$ and 0 for SFT respectively for ZIC2, ZIC3 and ZIC5).

\section{Supplementary Files}

This is a list of supplementary files associated with this preprint. Click to download.

- TFSSupplementaryFigure1 textcluster.txt

- TFSSupplementaryfigure1.png

- TFSSupplementarytables123revised.docx 